OPEN ACCESS

Edited by:

Zongxin Ling,

Zhejiang University, China

Reviewed by:

Sara Monteiro,

Centro Hospitalar de Trás os Montes

e Alto Douro, Portugal

Giuseppe Losurdo,

University of Bari Medical School, Italy

*Correspondence:

Bin Lu

drlvbin@163.com

tThese authors have contributed equally to this work

Specialty section

This article was submitted to

Gastroenterology,

a section of the journal

Frontiers in Medicine

Received: 12 September 2020 Accepted: 25 January 2021

Published: 15 February 2021

Citation:

Huang L, Hu Y, Chen F, Liu S and Lu B (2021) Effectiveness of Improved Use of Chewing Gum During Capsule Endoscopy in Decreasing Gastric

Transit Time: A Prospective

Randomized Controlled Study.

Front. Med. 8:605393.

doi: 10.3389/fmed.2021.605393

\section{Effectiveness of Improved Use of Chewing Gum During Capsule Endoscopy in Decreasing Gastric Transit Time: A Prospective Randomized Controlled Study}

\author{
Liang Huang ${ }^{1+}$, Yue $\mathrm{Hu}^{1 \dagger}$, Fang Chen ${ }^{2}$, Shan $\mathrm{Liu}^{1}$ and Bin $\mathrm{Lu}^{1 *}$ \\ ${ }^{1}$ First Affiliated Hospital, Zhejiang Chinese Medical University, Hangzhou, China, ${ }^{2}$ Department of Gastroenterology, \\ Hangzhou Red Cross Hospital, Hangzhou, China
}

Background/Aim: Chewing gum throughout small bowel capsule endoscopy (SBCE) increases completion rates (CRs) but decreases small bowel transit time (SBTT) and diagnostic yield (DY). We determined the effects of chewing gum early during SBCE on gastric transit time (GTT), SBTT, CR, DY, and gastroscopy intervention.

Methods: We prospectively enrolled patients (ages 16-80 years) undergoing SBCE between January and June 2019. Patients were randomized to a chewing gum group (103 patients) and a control group (102 patients). Patients in the former group chewed one piece of gum for $\sim 15$ min every 30 min during the first hour of SBCE. Two gastroenterologists blinded to the study group examined the data.

Results: GTT was shorter in the chewing gum group (29.0 min, interquartile range: $17.0-52.0 \mathrm{~min}$ ) than in the control group [ $42.5 \mathrm{~min}(23.25-60 \mathrm{~min}) ; P=0.01]$. SBTT was similar in the two groups [318.5 min (239.5-421.3 min) vs. $287.0 \mathrm{~min}$ (216.0-386.0 min); $P=0.08$ ]. Gastroscopy rate was lower in the chewing gum group (15.53 vs. $32.35 \%$, $P=0.005)$. CR (95.15 vs. $89.22 \%, P=0.114)$ and DY (67.96 vs. $59.80 \%, P=0.224)$ did not differ between the groups. The number of abnormal-lesion types detected per patient was higher in the chewing gum group [1.0 (0.0-2.0) vs. $2.0(0.0-2.0) ; P=0.049$ ].

Conclusions: Chewing gum early during SBCE significantly reduced GTT and gastroscopy intervention, with no influence on SBTT (Trial number: NCT03815136).

\footnotetext{
Keywords: small bowel capsule endoscopy, chewing gum, gastric transit time, small bowel transit time, gastroscopy intervention
}

\section{INTRODUCTION}

Small bowel capsule endoscopy (SBCE) has greatly facilitated the screening, diagnosis, and monitoring of small bowel diseases $(1,2)$. SBCE has been shown to be efficacious for conditions such as celiac disease, iron-deficiency anemia, small bowel tumors, and familial polyposis syndromes (3). It is especially useful as a first-line investigation for obscure gastrointestinal bleeding (4). The two most vital quality indicators for SBCE are considered to be its diagnostic yield (DY) and completion rate (CR) $(5,6)$. However, several factors can affect the DY and CR of SBCE, including 
small bowel preparation, gastrointestinal transit time, and the battery life of the capsule endoscope (3). A gastric transit time (GTT) $>45 \mathrm{~min}$ has been shown to be an independent risk factor for incomplete SBCE (7), while the DY of SBCE has been reported to be positively correlated with small bowel transit time (SBTT) (8). Moreover, gastric or esophageal retention of the capsule can prevent the endoscope from crossing the pylorus, prolonging GTT and even leading to a failure to reach the cecum within the available recording time. Furthermore, although the endoscopic placement of the capsule can be used to overcome this problem, this method increases patients' economic burden and discomfort (9).

The use of chewing gum throughout the SBCE examination simulates sham feeding, which shortens the GTT and/or SBTT and possibly increases the CR $(10,11)$. However, a decrease in SBTT may reduce the DY and therefore, the effectiveness of SBCE. We hypothesized that the limited use of chewing gum only in the first hour of the SBCE examination would shorten GTT but not SBTT and reduce gastroscopy intervention rate, and thereby improve the CR and DY of SBCE. The purpose of this study was to determine the effects of the limited use of chewing gum during SBCE on the GTT, SBTT, DY, CR and gastroscopy intervention of SBCE.

\section{METHODS}

\section{Study Design}

This prospective, endoscopist-blind, randomized, controlled pilot study enrolled consecutive patients who were scheduled to undergo SBCE at the Endoscopy Center of the First Affiliated Hospital, Zhejiang Chinese Medical University, between January and June 2019. The institutional review board approved the study protocol and informed consent form (IRB number 2019-K-199-01). This study has been registered at www.ClinicalTrials.gov (NCT03815136).

\section{Study Participants}

Patients aged 16-80 years who were scheduled to undergo SBCE and provided written informed consent were eligible to be included in this study. The exclusion criteria were as follows: previous abdominal surgery, diabetes mellitus, hyper- or hypothyroidism, use of prokinetics or narcotics within 5 days before SBCE, and refusal to participate in this study. In addition, patients with poor visibility on SBCE [visualization, $<75 \%$ of the mucosa (12)] were considered to have received poor bowel preparation and were excluded from the study.

Patients were randomly assigned to a chewing gum group or a control group at the time of making the appointment for SBCE. We used envelopes containing computer-generated random numbers generated by one of the investigators (L.H.) who was responsible for keeping the randomization key locked until the last patient had been enrolled. The patients were instructed not to tell the endoscopist or the investigators (H.Y. and C.F.) about whether or not they used chewing gum before, during, or after the SBCE procedure.

\section{Study Procedures}

All patients underwent identical bowel preparation prior to SBCE. The patients drank clear liquids for dinner on the day before capsule ingestion. On the day of the procedure, at 04:00-05:00 h, the patients drank two sachets of polyethylene glycol electrolyte powder dissolved in $2 \mathrm{~L}$ of water within a period of $2 \mathrm{~h}$. Each sachet contained $59 \mathrm{~g}$ polyethylene glycol $4,000,5.68 \mathrm{~g}$ sodium sulfate, $1.68 \mathrm{~g}$ sodium bicarbonate, $1.46 \mathrm{~g}$ sodium chloride, and $0.74 \mathrm{~g}$ potassium chloride (Hengkang Pharmaceutical Co, Jiangxi, China). In addition, the patients fasted overnight for at least $8 \mathrm{~h}$ before undergoing SBCE.

All examinations were commenced between 8:00 and 10:00 h. We used PillCam SB2 (Medtronic, Minnesota, America) for the examinations. Patients allocated to the chewing gum group were instructed to chew a piece of sugarless gum (Wrigley's Extra Sugar-Free Gum) for $\sim 15 \mathrm{~min}$ every $30 \mathrm{~min}$ during the first hour of the SBCE examination. Thus, each patient in this group chewed a total of 2 pieces of gum. The intervention was timed using an alarm. The patients allocated to the control group were not given chewing gum and were asked to refrain from doing a chewing movement.

All patients returned for review $\sim 60$ min later for realtime confirmation of whether the capsule camera had reached the small bowel. If it had not, the patient underwent gastric endoscopic placement of the capsule. The intake of solid foods was permitted $8 \mathrm{~h}$ after capsule ingestion. All patients returned to the SBCE recorder after $24 \mathrm{~h}$ of examination.

\section{Outcomes}

The primary outcomes were GTT and SBTT. GTT was defined as the interval between the first gastric and first duodenal images, while SBTT was defined as the interval between the first duodenal and first cecal images. The secondary outcomes were DY and CR. DY was defined as the rate of positive findings (diagnostic or suspicious) on SBCE examination, and $\mathrm{CR}$ was defined as the rate of complete recording, as indicated by the camera entering the cecum within the battery time. Capsule endoscopic finding per patient was sorted according to the following categories: normal variants (lymphangiectasia and lymphatic follicular hyperplasia) and abnormal lesions [ulcer, polyp, bleeding, vascular malformation, stenosis, diverticulum, parasite, tumor, and inflammation, which included inflammatory changes observed in the intestine during endoscopy (13), such as erosion, Crohn's disease, eosinophilic enteritis, intestinal tuberculosis, and radiation enteritis]. We also assessed the intervention rate, which was defined as the rate of endoscopic capsule placement in the duodenum when the GTT was over 60 $\min (9)$.

\section{Sample-Size Calculation}

Prior to the study, we calculated the sample size required based on our preliminary results, namely GTT (data shown in Supplementary Material). We determined that at least 182 patients were needed to detect significant differences on two-tailed tests with a 0.05 alpha level and $80 \%$ power. In our experience, approximately $10 \%$ of patients cancel their SBCE appointments or have bad bowel preparation. 


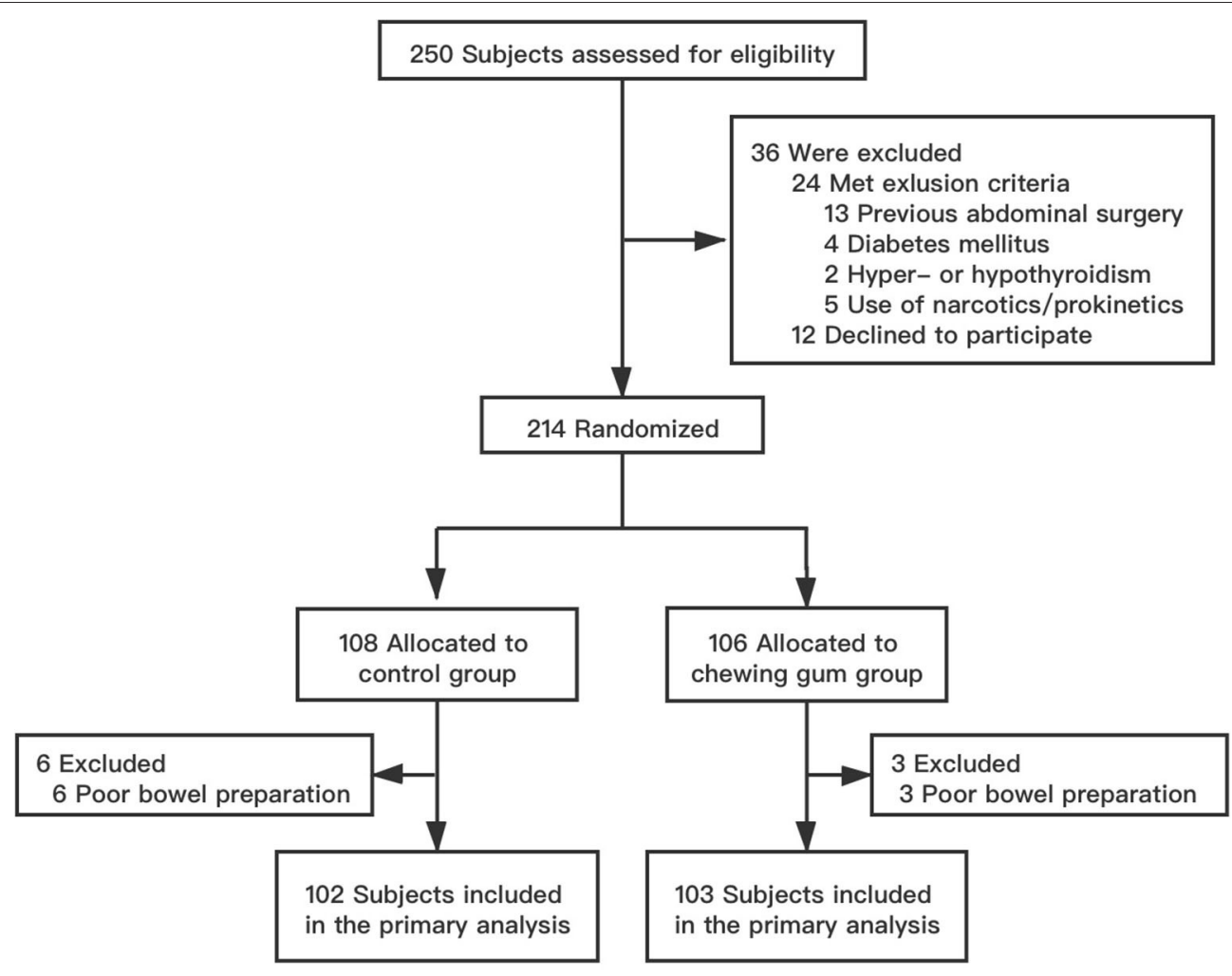

FIGURE 1 | Flowchart of the study.

Thus, a total sample size of 203 patients was estimated to be sufficient.

\section{Statistical Analysis}

Descriptive statistics were used for the baseline characteristics of the participants. Specifically, continuous variables with normal distribution were expressed as mean and SD (age and body-mass index), and compared using the Student $t$-test, while categorical variables were expressed as percentages and counts (gender and indication for capsule endoscopy), and compared using the $\chi^{2}$-test for categorical variables.

Between-group differences in outcomes were analyzed using the $\chi^{2}$-test in the case of categorical variables (DY, CR, and intervention rate) and the Mann-Whitney $U$-test of variance in the case of continuous variables with non-normal distribution (GTT, SBTT, and abnormal lesions per patient). GTT, SBTT, and abnormal lesion type per patient were expressed as medians and interquartile ranges ( $25 \mathrm{th}-75$ th percentiles). The hazard ratios for complete viewing of the stomach and small bowel in the control and chewing gum groups were assessed using Kaplan-Meier analysis (Log-rank). The statistical analysis was conducted using SPSS for Windows, version 23.0. All reported $P$-values were two-sided, and confidence intervals (CIs) were at the $95 \%$ level. Differences with $P<0.05$ were considered statistically significant.

\section{RESULTS}

\section{General Information}

Between January and June 2019, a total of 250 patients aged 1680 years were scheduled to undergo SBCE in our institution. Of these, 36 patients were excluded because they either did not meet the selection criteria (24 patients) or refused to participate in the study (12 patients). The remaining 214 patients were randomized to the control group (108 patients) and the chewing gum group (106 patients). Six patients from the control group and three patients from the chewing gum group were unable to undergo SBCE at the scheduled time because of poor bowel preparation $(P=0.514)$. Thus, the final analysis involved 102 subjects in the control group and 103 subjects in the chewing gum group. A flow chart of subject selection is shown in Figure 1. The baseline clinical characteristics were comparable between the two study groups (Table 1).

\section{Outcomes}

The outcomes of the study have been summarized in Table 2. The median GTT was significantly lower in the chewing gum group $(29.0 \mathrm{~min})$ than in the control group [ $42.5 \mathrm{~min}$; KaplanMeier: hazard ratio, 1.564; 95\% confidence interval (CI), 1.1372.153; $P=0.006$; Figure 2]. In contrast, the median SBTT did not differ between the chewing gum group (318.5 min) and control group (287.0 min; Kaplan-Meier: hazard ratio, 0.943; 95\% CI, $0.708-1.257 ; P=0.6913$; Figure 3). 
TABLE 1 | Characteristics of the study subjects.

\begin{tabular}{lccc}
\hline Parameter & $\begin{array}{c}\text { Control group } \\
(\boldsymbol{n}=\mathbf{1 0 2})\end{array}$ & $\begin{array}{c}\text { Chewing gum group } \\
(\boldsymbol{n}=\mathbf{1 0 3})\end{array}$ & $\boldsymbol{P}$-value \\
\hline Age, yrs, mean $\pm \mathrm{SD}$ & $45.00 \pm 15.60$ & $47.10 \pm 16.30$ & 0.347 \\
Gender & & & 0.953 \\
Female, $n(\%)$ & $43(42.16)$ & $43(41.75)$ & \\
Male, $n(\%)$ & $59(57.84)$ & $60(58.25)$ & \\
BMl, kg/m², mean $\pm \mathrm{SD}$ & $21.63 \pm 2.94$ & $21.92 \pm 3.85$ & 0.536 \\
Indication for capsule & & & 0.245 \\
endoscopy & & & \\
Screening, $n(\%)$ & $33(32.35)$ & $26(25.24)$ & \\
Diagnostic, $n$ (\%) & $65(63.73)$ & $68(66.02)$ & \\
Surveillance, $n$ (\%) & $4(3.92)$ & $9(8.73)$ & \\
& & &
\end{tabular}

BMI, body-mass index.

$P$-value: Student $t$-test for continuous variables and $x^{2}$-test for categorical data.

TABLE 2 | Effects of chewing gum on the outcomes of capsule endoscopy examination.

\begin{tabular}{lccc}
\hline Parameter & $\begin{array}{c}\text { Control group } \\
(\boldsymbol{n}=\mathbf{1 0 2})\end{array}$ & $\begin{array}{c}\text { Chewing gum group } \\
(\boldsymbol{n}=\mathbf{P} \text {-value }\end{array}$ & \\
\hline GTT, min, median (IQR) & $42.5(23.25,60.00)$ & $29.0(17.00,52.00)$ & 0.010 \\
SBTT, min, median (IQR) & $287.0(216.00,386.00)$ & $318.5(239.50,421.30)$ & 0.084 \\
Completion rate, $n(\%)$ & $91(89.22)$ & $98(95.15)$ & 0.114 \\
Diagnostic yield, $n$ (\%) & $61(59.80)$ & $70(67.96)$ & 0.224 \\
Abnormal lesions type, & $1(0.00,2.00)$ & $2(0.00,2.00)$ & 0.049 \\
$n$, median (IQR) & & & \\
Intervention rate, $n(\%)$ & $33(32.35)$ & $16(15.53)$ & 0.005 \\
\hline
\end{tabular}

GTT, gastric transit time; SBTT, small bowel transit time; IQR, interquartile range. $P$-value: Mann-Whitney U-test of variance for alphanumeric variables and $\chi^{2}$-test for categorical data.

The DY and CR also did not significantly differ between the chewing gum and control groups $(P=0.224$ and $P=0.114$, respectively). The number of abnormal small bowel-lesion types detected per patient was higher in the chewing gum group than in the control group $(P=0.049)$. The intervention (gastroscopy) rate was significantly lower in the chewing gum group than in the control group ( 15.53 vs. $32.35 \%, P=0.005)$.

\section{Findings and Incomplete Examinations}

The most frequent positive result on SBCE was gastrointestinal ulcer (control, 43.13\%; chewing gum, 44.66\%), followed by gastrointestinal inflammation (control, 21.57\%; chewing gum, $35.92 \%$ ) and small bowel stenosis (control, 9.80\%; chewing gum, $15.53 \%)$. Other SBCE findings included small bowel bleeding, small bowel vascular malformation, polyp syndrome, small bowel tumor, intestinal parasites, and intestinal diverticulum (Table 3).

SBCE could not be completed for 11 patients in the control group and five patients in the chewing gum group because of either capsule retention (due to carcinoma and ulcer) or battery power outage (Table 4). Among the nine patients with incomplete SBCE due to capsule retention, the following interventions were carried out: in one patient, the capsule was
A

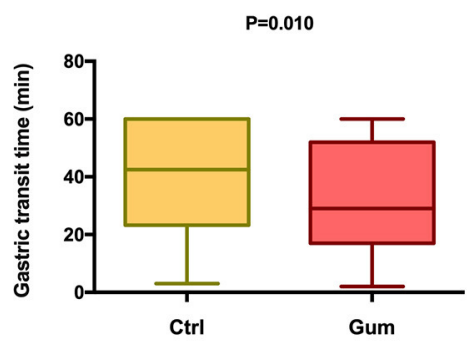

B

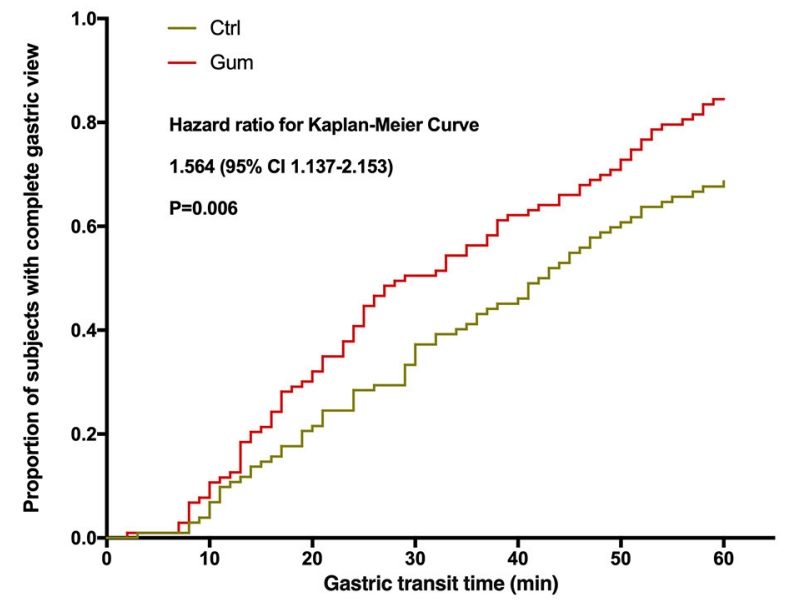

No. CE arrived to duodenum

$\begin{array}{llllllll}\text { Control } & 102 & 7 & 22 & 38 & 47 & 62 & 69\end{array}$
$\begin{array}{lllllll}\text { Chewing gum } 103 & 11 & 33 & 52 & 64 & 75 & 87\end{array}$

FIGURE 2 | Gastric transit time during capsule endoscopy in the chewing gum group and control group. (A) A boxplot with medians and quartiles; (B) Kaplan-Meier curves for time to complete gastric view.

excreted naturally after 7 days without any therapy; in two patients diagnosed with small intestinal carcinoma, the capsule was retrieved during surgical intervention; in two patients, the capsule was passed after medical therapy (at 1 month and 67 days); and in three patients, the capsule was retrieved using double-balloon enteroscopy.

\section{DISCUSSION}

The present study revealed that patients who chewed two pieces of gum during the first hour of the SBCE procedure had a significantly shorter GTT than control subjects who did no chewing movements during SBCE. The intervention (gastroscopy) rate was also significantly lower in the chewing gum group than in the control group. However, the SBTT, DY, and CR did not differ between the two groups. Interestingly, although the DY did not significantly differ between the two study groups, further analysis showed that the number of abnormal lesion type detected per patient was higher in the chewing gum group than in the control group. 
A

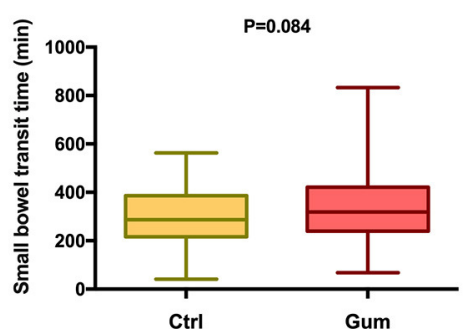

B

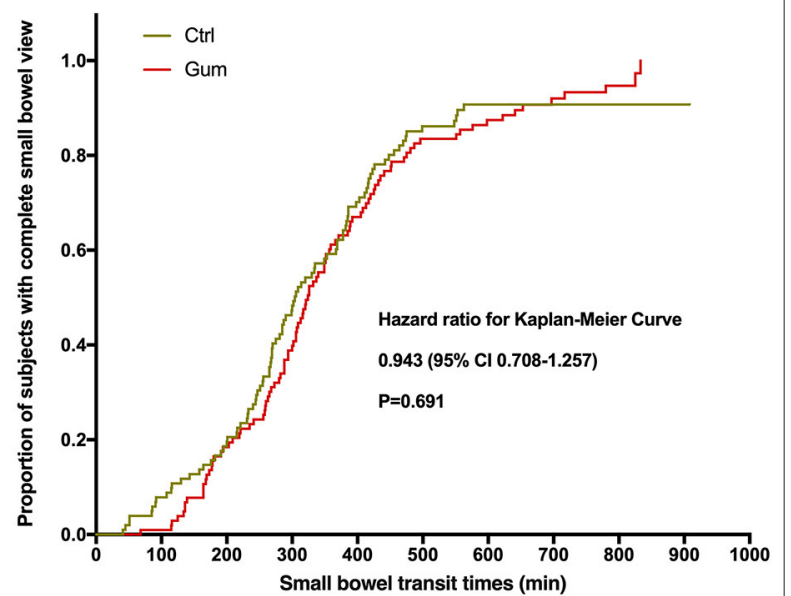

No. CE arrived to cecum

$\begin{array}{lllllllllll}\text { Control } & 91 & 8 & 20 & 49 & 71 & 87 & 91 & 91 & 91 & 91\end{array}$ $\begin{array}{lllllllllll}\text { Chewing gum } & 98 & 1 & 19 & 41 & 69 & 86 & 90 & 94 & 96 & 98\end{array}$

FIGURE 3 | Small bowel transit time during capsule endoscopy in the chewing gum group and control group. (A) A boxplot with medians and quartiles; (B) Kaplan-Meier curves for time to complete small bowel view.

Prokinetic drugs and metoclopramide have been used to shorten the GTT and/or SBTT and increase the CR of SBCE (14-16); however, these agents promote gastric as well as small intestinal movement throughout the examination period, and thus, limit the DY (4). Chewing gum is a safe, convenient, and inexpensive method of shortening the GTT via activation of vagal cholinergic stimulation of the bowel by simulating sham feeding $(17,18)$. However, studies on the effects of chewing gum on GTT, SBTT, and CR have reported mixed findings $(10,11,19)$. Consistent with our study, Apostolopoulos et al. (11) reported that the use of chewing gum during SBCE shortened the GTT. However, all of these previous studies used chewing gum throughout the SBCE examination, leading to a decrease in the SBTT and therefore the DY $(8,20)$. In contrast, chewing gum use was limited to the first hour of SBCE in our study, and we found that although the SBTT was longer in the chewing gum group than in the control group, the difference was not statistically significant. It is reasonable that chewing gum during the first hour of intervention did not increase the intestinal motility.

In our study, the CR did not differ between the two study groups. However, it should be noted that in most patients, the reason for the non-completion of the examination was capsule retention due to the presence of a lesion in the small intestine.
TABLE 3 | Findings of capsule endoscopy examination.

\begin{tabular}{|c|c|c|}
\hline Parameter & $\begin{array}{l}\text { Control group } \\
\qquad(n=102)\end{array}$ & $\begin{array}{l}\text { Chewing gum group } \\
\qquad(n=103)\end{array}$ \\
\hline \multicolumn{3}{|l|}{ Small-bowel abnormal lesions } \\
\hline Ulcer, $n(\%)$ & $44(43.13)$ & $46(44.66)$ \\
\hline Inflammation, $n(\%)$ & $22(21.57)$ & 37 (35.92) \\
\hline Polyps, $n(\%)$ & $7(6.86)$ & $4(3.88)$ \\
\hline Diverticulum, $n(\%)$ & None & $1(0.97)$ \\
\hline Parasite, $n(\%)$ & $1(0.98)$ & $5(4.85)$ \\
\hline Bleeding, $n(\%)$ & $4(3.92)$ & $8(7.77)$ \\
\hline Vascular malformation, $n(\%)$ & $2(1.96)$ & $9(8.74)$ \\
\hline Tumor, $n(\%)$ & $2(1.96)$ & $1(0.97)$ \\
\hline Stenosis, $n$ (\%) & $10(9.80)$ & $16(15.53)$ \\
\hline \multicolumn{3}{|l|}{ Normal variants } \\
\hline Lymphangiectasia, $n$ (\%) & $12(11.76)$ & $10(9.71)$ \\
\hline Lymphatic follicular hyperplasia, $n(\%)$ & $7(6.86)$ & $4(3.88)$ \\
\hline Normal, $n$ (\%) & 34 (33.33) & $25(24.27)$ \\
\hline
\end{tabular}

TABLE 4 | Reasons for non-completion of capsule endoscopy examination.

\begin{tabular}{lcc}
\hline Parameter & $\begin{array}{c}\text { Control group } \\
(\boldsymbol{n}=\mathbf{1 1})\end{array}$ & $\begin{array}{c}\text { Chewing gum group } \\
(\boldsymbol{n}=\mathbf{5})\end{array}$ \\
\hline Battery power outage, $n(\%)$ & $5(45.45)$ & $2(40.00)$ \\
Capsule retention, $n(\%)$ & $6(54.55)$ & $3(60.00)$ \\
Small intestinal stenosis, $n(\%)$ & $6(54.55)$ & $3(60.00)$ \\
Carcinoma, $n(\%)$ & $1(9.09)$ & $1(20.00)$ \\
Ulcer, $n(\%)$ & $5(45.45)$ & $2(40.00)$ \\
\hline
\end{tabular}

Shortening the GTT through chewing gum use is not expected to have an effect on the completion of the examination in such patients. Moreover, compared to the control group, the chewing gum group showed a higher number of abnormal lesions categories for per patient, which may theoretically lead to an increase in capsule retention rates, though no statistical difference was found in the present study. It should however be noted that the DY did not significantly differ between the chewing gum and control groups. There are several potential reasons for the lack of an increase in the SBTT, DY, and CR in our study. First, our study included both participants with normal findings on SBCE as well as participants with diverse clinical conditions; this could have limited the DY after randomization. Second, intestinal motility may have differed between the two study groups, which may have contributed to the lack of significant differences in the above parameters. Third, the sample size was small, and was calculated based on GTT before this trial, which might explain the negative results. Finally, the gastroscopy intervention in the first hour would have reduced the differences in SBTT, DY, and CR between the two study groups by reducing the original GTT.

In this study, the gastroscopy intervention rate was significantly lower in the chewing gum group than in the control group. This indicated that the use of chewing gum during the 
first hour of the examination could enhance gastrointestinal motility and accelerate the transit of the capsule endoscope through the stomach. In our clinic, we usually check the position of the capsule endoscope at $30 \mathrm{~min}$ after swallowing, and perform some intervention (mainly gastroscopy) if the capsule fails to pass through the stomach at $1 \mathrm{~h}$ after swallowing. The decrease in the gastroscopy rate indicated that the use of chewing gum early during the procedure could reduce human intervention during SBCE, especially in terms of gastroscopy, which can cause discomfort to patients. In addition, Buijs et al. (21) reported that chewing two pieces of sugar-free chewing gum after the capsule left the stomach could increase the excretion rate (not significantly), which might improve the bowel-cleansing quality. However, we compared the rate of poor bowel preparation between the two groups, and found no significant difference in this rate $(5.56$ vs. $2.83 \%, P=0.514)$. In our study, the chewing gum intervention was mainly restricted to the period when the capsule was in the stomach in the first hour of the examination, which might explain the relatively limited effect on bowel preparation. Previous studies indicated that capsule endoscopy has a good diagnostic yield rate in iron-deficiency anemia patients, about $47 \%$ (22). In particular, more vascular (31 vs. $22.6 \%, P=0.007)$, inflammatory ( 17.8 vs. $11.3 \%, P=$ 0.009 ), and mass/tumor (7.95 vs. $2.25 \%, P=0.0001)$ lesions were detected with SBCE (22). Contaldo et al. (23) showed that VCE could reveal the source of obscure-occult bleeding in a high percentage of unexplained iron-deficiency anemia. However, our result did not find the validity of SBCE in the investigation of patients with IDA and negative findings on a previous diagnostic workup.

The current study has a few limitations. First, this was a single-center study, which may have affected the outcomes due to the bias of the collected data. Second, there is no true gold standard test against which SBCE may be compared, and therefore, there may be an underlying rate of missed cases that cannot be assessed. However, as the repeatability of SBCE in the same patients may be objective and reliable, and this can be used to reduce bias to a certain extent in future studies. Finally, the higher detection rate of abnormallesion types in the chewing gum group as compared to the control group was on the borderline of statistical significance $(P=0.049)$. We therefore performed logistic regression analysis to evaluate the effects of age, sex, body-mass index, and number of abnormal-lesion types. Among these factors, only the number of abnormal-lesion types was found to be significant $(P=0.034$, odds ratio $=1.358,95 \%$ confidence interval: 1.024-1.801). This result lends further support to the study findings. In the future, more high-quality, large-scale studies will help overcome these limitations and draw more solid conclusions.

\section{REFERENCES}

1. Pennazio M, Spada C, Eliakim R, Keuchel M, May A, Mulder CJ, et al. Small-bowel capsule endoscopy and device-assisted enteroscopy for diagnosis and treatment of small-bowel disorders: european Society of
In summary, the limited use of chewing gum during the first hour of SBCE significantly reduced the GTT but not the SBTT. The use of chewing gum did not reduce the CR of SBCE and significantly reduced the gastroscopy intervention rate. We believe that the use of chewing gum during the first hour of SBCE might improve the detection of abnormal lesions, as the SBTT remains unaltered during the procedure.

\section{DATA AVAILABILITY STATEMENT}

The original contributions presented in the study are included in the article/Supplementary Material, further inquiries can be directed to the corresponding author/s.

\section{ETHICS STATEMENT}

The studies involving human participants were reviewed and approved by The institutional review board approved the study protocol and informed consent form (the First Affiliated Hospital, Zhejiang Chinese Medical University;IRB number 2019-K-199-01). Written informed consent to participate in this study was provided by the participants' legal guardian/next of kin.

\section{AUTHOR CONTRIBUTIONS}

BL designed and supervised the study, including all data collection and analysis. LH, YH, and FC performed most of the investigations, including data collection and analysis, and wrote the manuscript. SL provided guidance on data analysis. All authors have read and approved the manuscript.

\section{FUNDING}

This study was supported by the Zhejiang Administration of Traditional Chinese Medicine (Grant No., 2020ZA051).

\section{ACKNOWLEDGMENTS}

This study was presented in part at the Clinical Endoscopic Practice Topic Forum at Digestive Diseases Week (San Diego) in May 2019.

\section{SUPPLEMENTARY MATERIAL}

The Supplementary Material for this article can be found online at: https://www.frontiersin.org/articles/10.3389/fmed. 2021.605393/full\#supplementary-material

Excel Sheet 1 | Original data.

Excel Sheet 2 | Original data of pre-experiment.

Gastrointestinal Endoscopy (ESGE) Clinical Guideline. Endoscopy. (2015) 47:352-76. doi: 10.1055/s-0034-1391855

2. Enns RA, Hookey L, Armstrong D, Bernstein CN, Heitman SJ, Teshima C, et al. Clinical practice guidelines for the use of video capsule endoscopy. Gastroenterology. (2017) 152:497-514. doi: 10.1053/j.gastro.2016.12.032 
3. Ladas SD, Triantafyllou K, Spada C, Riccioni ME, Rey JF, Niv Y, et al. European Society of Gastrointestinal Endoscopy (ESGE): recommendations (2009) on clinical use of video capsule endoscopy to investigate small-bowel, esophageal and colonic diseases. Endoscopy. (2010) 42:220-7. doi: 10.1055/s-0029-1243968

4. Gerson LB, Fidler JL, Cave DR, Leighton JA. ACG clinical guideline: diagnosis and management of small bowel bleeding. Am J Gastroenterol. (2015) 110:1265-87. doi: 10.1038/ajg.2015.246

5. Koulaouzidis A, Giannakou A, Yung DE, Dabos KJ, Plevris JN. Do prokinetics influence the completion rate in small-bowel capsule endoscopy? A systematic review and meta-analysis. Curr Med Res Opin. (2013) 29:117185. doi: 10.1185/03007995.2013.818532

6. Liao Z, Xu C, Li ZS. Completion rate and diagnostic yield of small-bowel capsule endoscopy: 1 vs. 2 frames per second. Endoscopy. (2010) 42:3604. doi: 10.1055/s-0029-1243993

7. Westerhof J, Weersma RK, Koornstra JJ. Risk factors for incomplete small-bowel capsule endoscopy. Gastrointest Endosc. (2009) 69:74-80. doi: 10.1016/j.gie.2008.04.034

8. Westerhof J, Koornstra JJ, Hoedemaker RA, Sluiter WJ, Kleibeuker JH, Weersma RK. Diagnostic yield of small bowel capsule endoscopy depends on the small bowel transit time. World J Gastroenterol. (2012) 18:15027. doi: 10.3748/wjg.v18.i13.1502

9. Gao YJ, Ge ZZ, Chen HY, Li XB, Dai J, Ye CA, et al. Endoscopic capsule placement improves the completion rate of small-bowel capsule endoscopy and increases diagnostic yield. Gastrointest Endosc. (2010) 72:1038. doi: 10.1016/j.gie.2009.12.003

10. Lam TT, Keegan AD. Chewing gum shortens small bowel but not gastric transit time during capsule endoscopy. Gastroenterology. (2010) 138(Suppl 1):S669. doi: 10.1016/S0016-5085(10)63077-6

11. Apostolopoulos P, Kalantzis C, Gralnek IM, Liatsos C, Tsironis C, Kalantzis N. Clinical trial: effectiveness of chewing-gum in accelerating capsule endoscopy transit time-a prospective randomized, controlled pilot study. Aliment Pharmacol Ther. (2008) 28:405-11. doi: 10.1111/j.1365-2036.2008.03762.x

12. van Tuyl SA, den Ouden H, Stolk MF, Kuipers EJ. Optimal preparation for video capsule endoscopy: a prospective, randomized, single-blind study. Endoscopy. (2007) 39:1037-40. doi: 10.1055/s-2007-966988

13. Lim YJ, Lee OY, Jeen YT, Lim CY, Cheung DY, Cheon JH, et al. Indications for detection, completion, and retention rates of small bowel capsule endoscopy based on the 10-year data from the Korean capsule endoscopy registry. Clin Endosc. (2015) 48:399-404. doi: 10.5946/ce.2015.48.5.399

14. Almeida N, Figueiredo P, Freire P, Lopes S, Lérias C, Gouveia H, et al. The effect of metoclopramide in capsule enteroscopy. Dig Dis Sci. (2010) 55:153-7. doi: 10.1007/s10620-008-0687-y
15. Wei W, Ge ZZ, Lu H, Gao YJ, Hu YB, Xiao SD. Effect of mosapride on gastrointestinal transit time and diagnostic yield of capsule endoscopy. J Gastroenterol Hepatol. (2007) 22:16058. doi: 10.1111/j.1440-1746.2007.05064.x

16. Caddy GR, Moran L, Chong AK, Miller AM, Taylor AC, Desmond PV. The effect of erythromycin on video capsule endoscopy intestinal-transit time. Gastrointest Endosc. (2006) 63:262-6. doi: 10.1016/j.gie.2005.07.043

17. McCormick JT. Gum in the postoperative setting: something to chew on. Dis Colon Rectum. (2013) 56:273-4. doi: 10.1097/DCR.0b013e31827d1605

18. Jiang Z, Liang H, Huang Z, Tang J, Tang L. Sham feeding with chewing gum in early stage of acute pancreatitis: a randomized clinical trial. Med Sci Monit. (2017) 23:623-30. doi: 10.12659/MSM.903132

19. Ou G, Svarta S, Chan C, Galorport C, Qian H, Enns R. The effect of chewing gum on small-bowel transit time in capsule endoscopy: a prospective, randomized trial. Gastrointest Endosc. (2014) 79:6306. doi: 10.1016/j.gie.2013.08.038

20. Buscaglia JM, Kapoor S, Clarke JO, Bucobo JC, Giday SA, Magno P, et al. Enhanced diagnostic yield with prolonged small bowel transit time during capsule endoscopy. Int J Med Sci. (2008) 5:303-8. doi: 10.7150/ijms.5.303

21. Buijs MM, Kobaek-Larsen M, Kaalby L, Baatrup G. Can coffee or chewing gum decrease transit times in Colon capsule endoscopy? A randomized controlled trial. BMC Gastroenterol. (2018) 18:95. doi: 10.1186/s12876-018-0824-9

22. Koulaouzidis A, Rondonotti E, Giannakou A, Plevris JN. Diagnostic yield of small-bowel capsule endoscopy in patients with irondeficiency anemia: a systematic review. Gastrointest Endosc. (2012) 76:983-92. doi: 10.1016/j.gie.2012.07.035

23. Contaldo A, Losurdo G, Albano F, Iannone A, Barone M, Ierardi E, et al. The spectrum of small intestinal lesions in patients with unexplained iron deficiency anemia detected by video capsule endoscopy. Medicina (Kaunas). (2019) 55:59. doi: 10.3390/medicina55030059

Conflict of Interest: The authors declare that the research was conducted in the absence of any commercial or financial relationships that could be construed as a potential conflict of interest.

Copyright (c) 2021 Huang, Hu, Chen, Liu and Lu. This is an open-access article distributed under the terms of the Creative Commons Attribution License (CC BY).

The use, distribution or reproduction in other forums is permitted, provided the original author(s) and the copyright owner(s) are credited and that the original publication in this journal is cited, in accordance with accepted academic practice. No use, distribution or reproduction is permitted which does not comply with these terms. 